\title{
Ungewöhnlicher Verlauf einer Strahlenpneumonitis mit nachfolgender Bronchiolitis obliterans mit organisierender Pneumonie (BOOP) bei einer Patientin mit Mammakarzinom
}

\author{
D. Heinrichs ${ }^{1}$ \\ M. Amthor ${ }^{2}$ \\ D. Kirsten ${ }^{1}$
}

\author{
Uncommon Course of Radiation-Induced Pneumonitis with Subsequent \\ Bronchiolitis Obliterans with Organizing Pneumonia (BOOP) in a Female with \\ Breast Cancer
}

\section{Zusammenfassung}

Strahlenpneumonien nach Radiatio wegen Mamma-Karzinom sind eine bekannte Komplikation dieser Therapie. Das Auftreten einer BOOP nach Strahlentherapie eines Mammakarzinoms ist jedoch extrem selten und wird kaum in die Differenzialdiagnose einbezogen. Die Folge davon sind große diagnostische Verschleppungszeiten und eine zu spät einsetzende Therapie. Die Tatsache, dass oft die nicht bestrahlte Lunge die chronische Pneumonie aufweist, ist ein erschwerender Faktor bei der Diagnostik. Anhand eines charakteristischen Krankheitsfalles soll auf die durch Bestrahlung ausgelöste BOOP-Reaktion, die auch nach einer vorangegangenen Strahlenpneumonitis auftreten kann, hingewiesen werden.

\section{Abstract}

Radiation-induced pneumonitis is a familiar complication of breast cancer radiotherapy, whereas bronchiolitis obliterans with organizing pneumonia occurs extremely rarely after this treatment. Consequently, testing for BOOP is rarely included in differential diagnosis. This results in delayed diagnosis, so that treatment is ultimately commenced too late. The infiltrations are often located in the unirradiated lung, impeding diagnosis. The radiotherapy-induced BOOP-reaction that can also occur after typical radiation pneumonitis is illustrated by reference to a characteristic case report.

\section{Einleitung}

Das Auftreten einer Strahlenpneumonitis nach Bestrahlungstherapie bei Mammakarzinom ist allgemein bekannt. Hier gibt es meist einen klaren zeitlichen Zusammenhang von Bestrahlung und dem Auftreten von flächigen Lungeninfiltraten. Eine BOOP dagegen tritt bei den unterschiedlichsten Grunderkrankungen auch nach einer Strahlentherapie eines Tumorleidens - als Komplikation auf. Beim Fehlen einer Grunderkrankung wird heute der Terminus COP (cryptogenic organizing pneumonia) gewählt.
Das Auftreten einer BOOP nach einer Strahlentherapie wegen eines Mammakarzinoms ist eine sehr seltene Komplikation dieser Therapie [1]. Unsere Publikation soll auf diesen Zusammenhang aufmerksam machen, da sicher nicht nur international die diagnostischen Verschleppungszeiten beachtlich sind.

Die „klassische“ Strahlenpneumonitis tritt bei Patientinnen auf, die aufgrund pulmonaler oder nicht-pulmonaler maligner Erkrankungen bestrahlt wurden [2,3]. Die meisten radiologischen Veränderungen werden im Gebiet der bestrahlten Lungenregion beobachtet. Es werden jedoch auch immer wieder Infiltrationen auf der nicht-bestrahlten Lunge beobachtet [4].

Institutsangaben

${ }^{1}$ Krankenhaus Großhansdorf. Zentrum für Pneumologie und Thoraxchirurgie

2Pathologisches Institut des Diakonissenkrankenhauses Rotenburg/Wümme

Anmerkung

Herrn Prof. Dr. H. Magnussen zum 60. Geburtstag gewidmet.

Korrespondenzadresse

Dr. D. Heinrichs · Krankenhaus Großhansdorf · Zentrum für Pneumologie und Thoraxchirurgie . Wöhrendamm $80 \cdot 22927$ Großhansdorf ·E-mail: heinrichs_dirk@hotmail.com

Eingang: 7. April 2004 · Nach Revision alkzeptiert: 5. Mai 2004

Bibliografie

Pneumologie 2004; 58: 404-407 · @ Georg Thieme Verlag KG Stuttgart · New York DOI $10.1055 / \mathrm{s}-2004-818482$

ISSN 0934-8387 
Bei Untersuchungen mit der bronchoalveolären Lavage (BAL) bei bestrahlten Patientinnen konnten lymphozytäre Alveolitiden auch in der unbestrahlten Lunge diagnostiziert werden $[5,6]$, obwohl seitens der Strahlentherapie-Planung versucht wird die "gesunde Seite“ zu schonen. Tangentiale Strahlenwirkungen geringen Umfangs werden aber immer die nicht-affektierte Seite erreichen. Die Art des Lungenparenchymschadens - akut/chronisch - ist von mehreren Einflussgrößen abhängig. Zu nennen sind Strahlengesamtdosis, Einzeldosengröße, Applikationsintervalle, Strahlenart und Größe des Lungenvolumens, das in das Strahlenfeld einbezogen werden muss. Das Krankheitsbild der Strahlenpneumonitis umfasst: die akute Pneumonie mit exsudativer Phase, gefolgt von einer organisierenden Phase mit Akkumulation von Entzündungszellen im Lungeninterstitium, meist
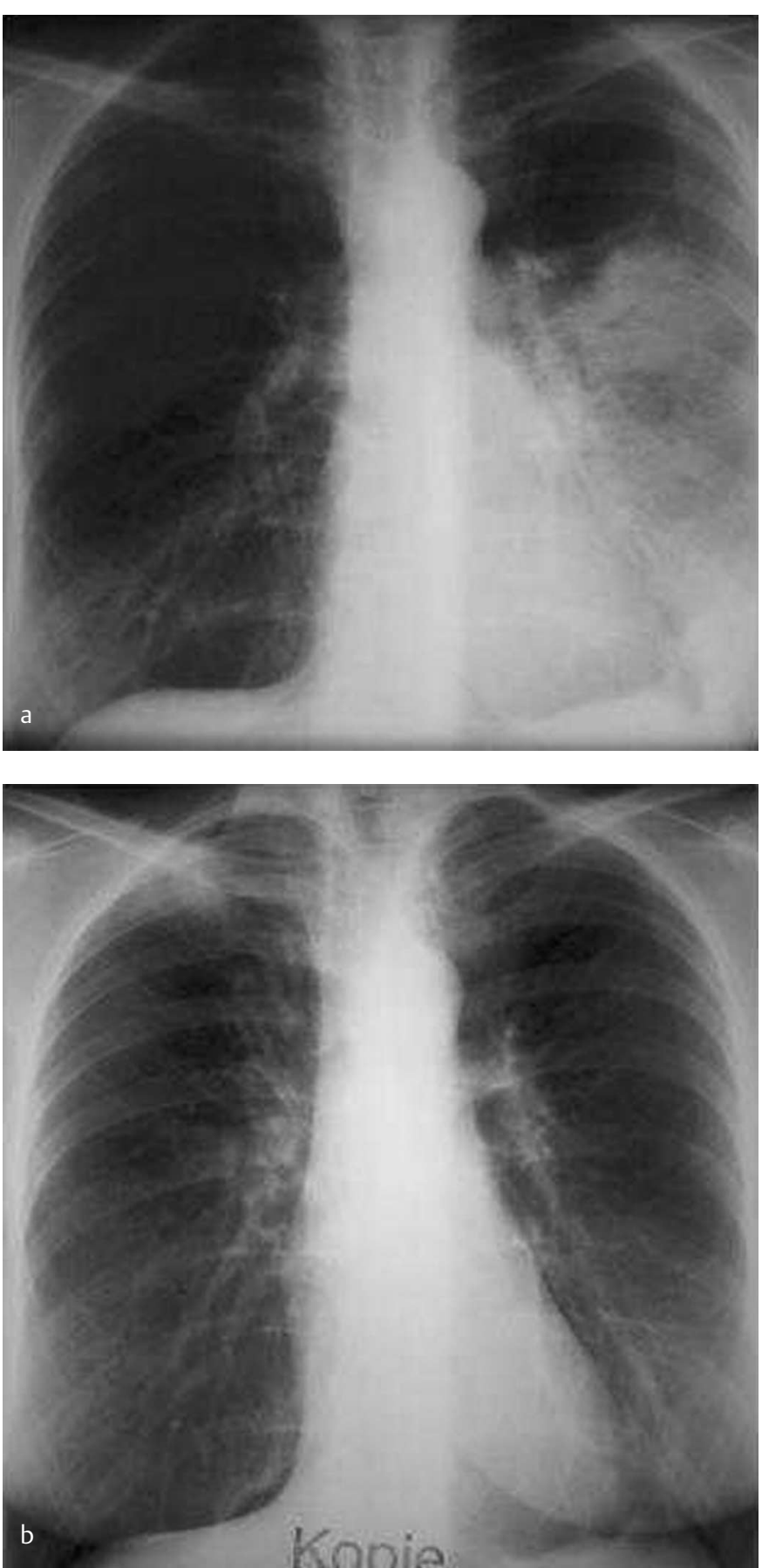

neutrophiler Granulozyten. Die chronische Strahlenpneumonie ist durch progredienten fibrotischen Umbau geprägt.

Während die Strahlenpneumonitis eine bekannte Komplikation darstellt, bedeuten wechselnde - auch in der nicht bestrahlten Lungenregion auftretende Infiltrate - diagnostische Probleme [1]. Transbronchiale Biopsien aus diesen Infiltraten ergeben histologische Bilder einer BOOP-Reaktion mit Granulationsgewebspfröpfen und proliferierten Fibroblasten in einem polysaccharidreichen Stroma im Gebiet der terminalen Bronchioli und der Alveolen. Eine milde begleitende lymphozytäre Alveolitis, selten auch Eosinophilie wird beobachtet [7].

Histologische Befunde mit hyalinen Membranen, Riesenzellen, Thromben und vaskulitischen Veränderungen, wie sie bei der Strahlenpneumonie entstehen, treten bei der BOOP nach Radiatio nicht auf.

\section{Fallbericht}

\section{Anamnese}

Bei einer 60-jährigen Patientin wurde zwei Jahre zuvor ein invasives Karzinom am Übergang unterer innerer/unterer äußerer Quadrant der linken Mamma brusterhaltend reseziert. Nach adjuvanter Chemotherapie (6 Zyklen CMF) erfolgte die konsolidierende Strahlenbehandlung zunächst der gesamten linken Mamma mit $4 \mathrm{MeV}$ Photonen bis $50 \mathrm{~Gy}$ sowie anschließender Dosisaufsättigung der ehemaligen Tumorregion mit $10 \mathrm{MeV}$ Elektronen bis $10 \mathrm{~Gy}$. Drei Monate später klagte die Patientin langsam zunehmend über Dyspnoe und vermehrte Müdigkeit. Die daraufhin veranlasste Röntgen-Thorax-Aufnahme (Abb.1a) zeigte eine ausgedehnte fleckige Verschattung betont in Projektion auf die Lingula mit positivem Luftbronchogramm bei unauffälliger Darstellung der Pleura. Unter der Diagnose einer Strahlenpneu-

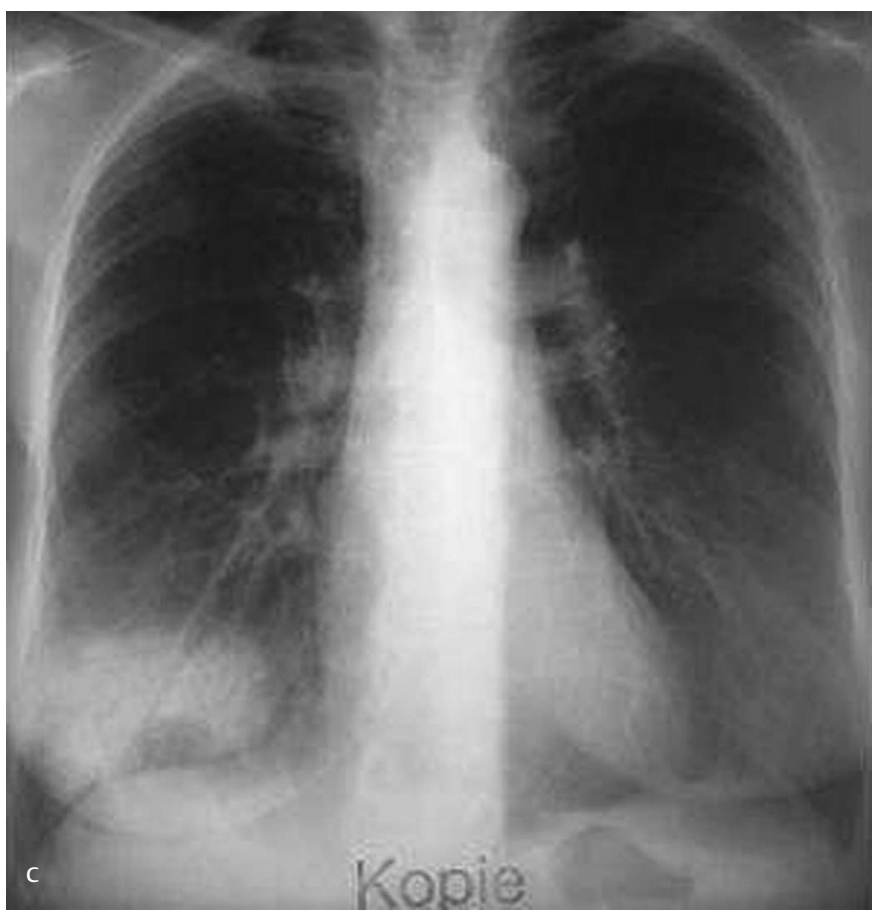

Abb. 1 a-c Wechselnde Lungeninfiltrate im Verlauf der strahleninduzierten BOOP. 
monitis erfolgte eine Prednisolontherapie über 6 Monate. Im Verlauf kam es sowohl klinisch als auch radiologisch zu einer vorübergehenden Besserung. Zum Ende der ausschleichenden Therapie traten dann erneut die Beschwerden auf, radiologisch wurde ein neues Infiltrat, jetzt im rechten Oberlappen erfasst (Abb. 1b). Unter Gabe von $25 \mathrm{mg}$ Prednisolon über ca. 4 Monate langsam ausschleichend waren Beschwerden und Infiltrat wieder rückläufig. Wiederum ein Monat später ergab sich eine neue Infiltration in einem anderen Segment des rechten Oberlappens und wiederum zwei Monate später im rechten Unterlappen (Abb.1c). Bei der nun veranlassten Krankenhausaufnahme beklagte die Patientin allgemeine Abgeschlagenheit, Dyspnoe und unproduktiven Husten.

\section{Aktuelle Befunde}

Die körperliche Untersuchung ergab eine Klopfschallverkürzung sowie inspiratorisches Knisterrasseln rechts basal bei vesikulärem Atemgeräusch, im Routinelabor eine BSG-Beschleunigung auf $61 \mathrm{~mm} /$ Std. sowie leicht erhöhte Leberparameter. Radiologisch zeigte sich ein flaues Restinfiltrat in Projektion auf den rechten Oberlappen sowie ein Unterlappeninfiltrat der gleichen Seite. Entsprechend wiesen computertomographisch die Segmente 7, 8 und 9 rechts eine ausgedehnte alveoläre Konsolidierung mit positivem Bronchopneumogramm und umgebender milchglasartiger Trübung auf(Abb. 2). In Segment 1 rechts fand sich eine keilförmige, peripher breitbasig aufsitzende Verdichtungszone. Vereinzelt erkannte man kleinste Bronchiektasen. Die Lungenfunktionsparameter lagen im Referenzbereich. Die Diffusionskapazität war mittelgradig eingeschränkt. Bronchoskopisch ergab sich zunächst kein Befund von Krankheitswert, lediglich bei der bronchoalveolären Lavage ließen sich bis zur letzten Portion Sekretausgüsse aspirieren. Das Zellbild wies eine nur leichtgradige Lymphozytose (18\%) bei sehr niedriger Gesamtzellzahl auf.

Die transbronchiale Zangenbiopsie aus dem rechten Unterlappen erfasste mikroskopisch Bronchusschleimhaut, äußere Bronchuswandanteile sowie alveoläres Parenchym. Die alveoläre Architektur war erhalten. In einzelnen Alveolen fanden sich als Zeichen der Mangelbelüftung reichlich Schaumzellen (Abb. 3). Intraluminal zeigten sich wiederholt zellreiche, zum Teil auch verzweigte Bindegewebspfröpfe (Abb.4), welche z.T. den hier luminal verbreiterten Septen anhingen und z.T. von Epithel bedeckt waren, so dass hier in der Gesamtschau das Bild einer BOOP bestand. Die Patientin wurde mit einer Prednisolontagesdosis von $40 \mathrm{mg}$ entlassen. Aufgrund des bisherigen Verlaufes wurde die Dosis nur langsam auf zuletzt $5 \mathrm{mg}$ reduziert. Ein Auslassversuch steht aus.
Seit nunmehr $1 \frac{1}{2}$ Jahren bestehen Rezidivfreiheit und Wohlbefinden.

\section{Diskussion}

Der Mechanismus der Entstehung einer BOOP-Reaktion nach Strahlentherapie von Mamma-Tumoren ist nicht vollends geklärt. Die Entstehung einer diffusen lymphozytären Alveolitis ist sicher nicht der einzige Weg. Allerdings erklärt die bereits oben erwähnte prospektive Studie von Martin und Mitarb. [6], bei der Patientinnen mit Mammakarzinom vor und zweimal nach ihrer Bestrahlung lavagiert wurden, die Beidseitigkeit der Befunde. Bei 22 von 26 Patientinnen konnte eine Alveolitis gefunden werden, allerdings entwickelten nur 8 (31\%) radiologische Infiltrate 15-90 Tage nach Beendigung der Strahlentherapie.

Die BAL-Befunde sprechen für ein so genanntes priming der Lymphozyten durch die Radiatio. Dass nur ein kleiner Teil der bestrahlten Patientinnen - trotz gleichen Strahlendosen - eine BOOP entwickelt, lässt auf einen individuellen Transkriptionsoder Zytokin-Polymorphismus [8] schließen, der eine derartig gesteigerte Susceptibilität hervorrufen kann [7].

Bei unserer Patientin ging der Strahlentherapie eine Chemotherapie mit Cyclophosphamid, Methotrexat und 5- Fluorouracil voraus. Grundsätzlich ist auch eine Chemotherapie bei Patientinnen mit Mammakarzinom in der Lage eine BOOP-Reaktion hervorzurufen. Bei einer Nachuntersuchung von 157 nachbestrahlten Patientinnen in Japan fand sich jedoch kein Unterschied in der Häufigkeit der BOOP-Entwicklung nach Tamoxifen und/oder Chemotherapie [9]. Diese spezielle Strahlenkomplikation ist sicherlich zu selten - in dieser Arbeit mit 2,5\% beziffert - um Hinweise auf die anzunehmende zusätzliche Sensibilisierung durch die Chemotherapie zu geben.

Das Auftreten von Lungeninfiltraten - gerade auch ihr Vorhandensein in der unbestrahlten Lunge - führt oft zu diagnostischen Fehlschlüssen mit der Folge längerer erfolgloser antibiotischer Behandlungen.

Die klinische Symptomatologie der Patientinnen ist uneinheitlich. Falls Fieber auftritt wird in aller Regel an eine infektiöse Ursache gedacht. Meist besteht eine erhebliche allgemeine Mattigkeit und Kraftlosigkeit [10].

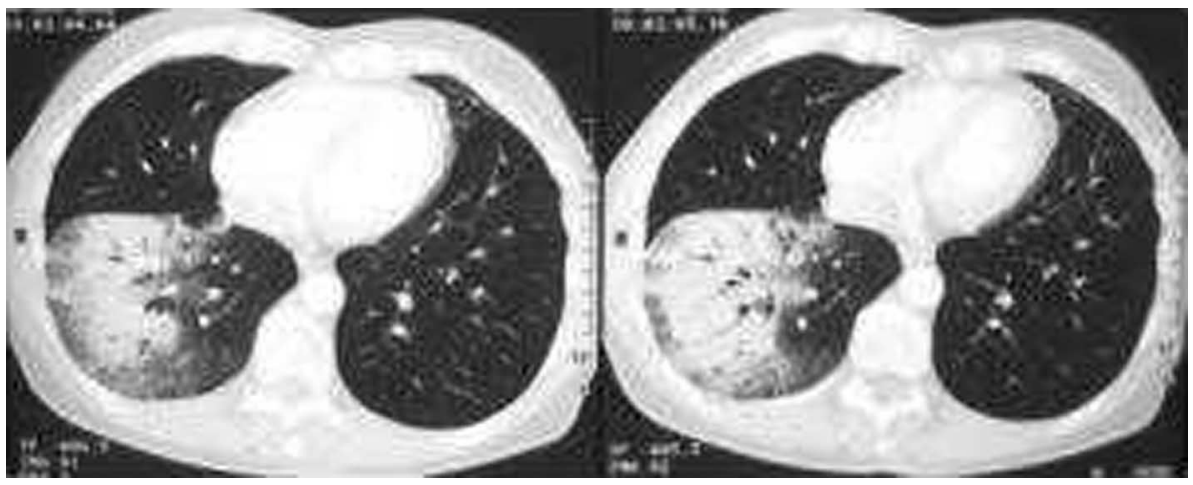

Abb 2 Computertomographie: Alveoläre Konsolidierung des rechten Lungenunterlappens vor Therapie. 


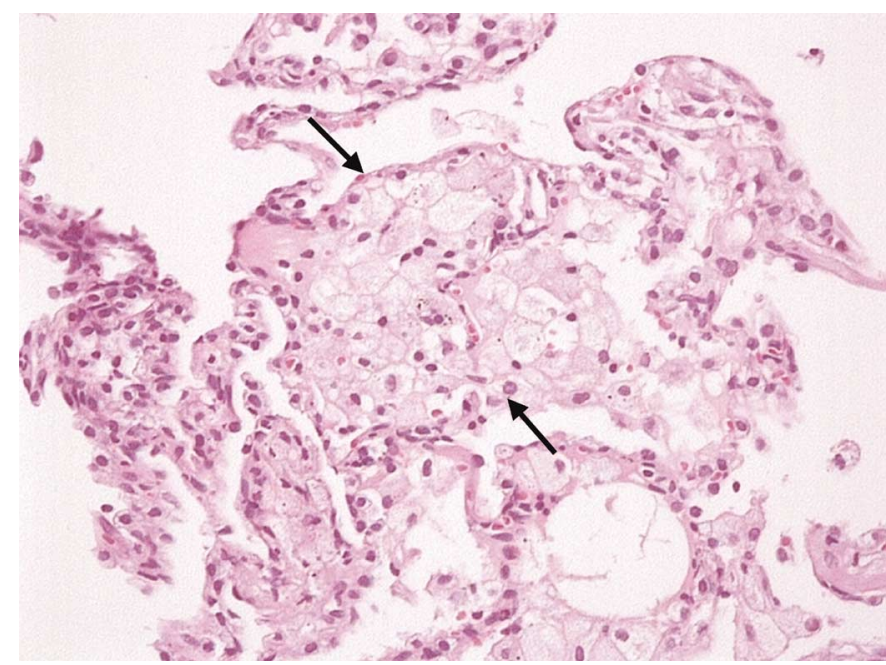

Abb 3 Alveole mit Schaumzellen als Zeichen einer Mangelbelüftung, HE-Färbung 100-fach.

In der Literatur wird die diagnostische Latenz zwischen ersten klaren Symptomen und der finalen Diagnose im Mittel auf 13-14 Wochen beziffert [10]. Der längste uns bekannte Beobachtungszeitraum ging über nahezu 10 Jahre. Eine unserer weiteren Patientinnen wurde 1983 operiert und nachbestrahlt. 1984 erfolgte eine Kortisontherapie bei Strahlenpneumonitis. Im Anschluss daran kam es zu Phasen mit Verschlechterung des Allgemeinzustands ohne Fieber mit dann röntgenologisch mehrfach nachgewiesenen Infiltraten. Wiederholt wurden wegen des fehlenden Ansprechens auf die Antibiotikagabe Pilzpneumonien diskutiert. Die Diagnose wurde erst im Februar 2003 mittels transbronchialer Lungenbiopsie gesichert.

Neben den Patientinnen mit Mammakarzinom sahen wir in den letzten Monaten BOOP-Komplikationen nach adjuvanter Radiatio bei Schilddrüsen- sowie bei Bronchialkarzinomen. Sowohl der klinische Verlauf als auch das computertomographische Bild war z.T. so typisch, dass auf die diagnostische Sicherung mittels Lungenbiopsie verzichtet werden konnte.

Die Therapie umfasst Kortison in einer Dosierung zwischen $20-50 \mathrm{mg} / \mathrm{d}$. Die Dosisreduktion kann nach 14 Tagen erfolgen, wenn klinisch und radiologisch eine Besserung zu erzielen war. Die weitere Dosierung sollte dem klinischen Befund und den Röntgenbefunden angepasst werden. Eine Mindestbehandlungszeit von 6 Monaten in Anlehnung an die Behandlungsstrategien anderer BOOP-Fälle darf möglichst nicht unterschritten werden. Rezidive sind beschrieben und in erster Linie, wie der oben geschilderte Fall zeigt, zu kurzen Therapiezeiten zuzuschreiben. Wir behandelten unsere Patientinnen über mindestens 9-12

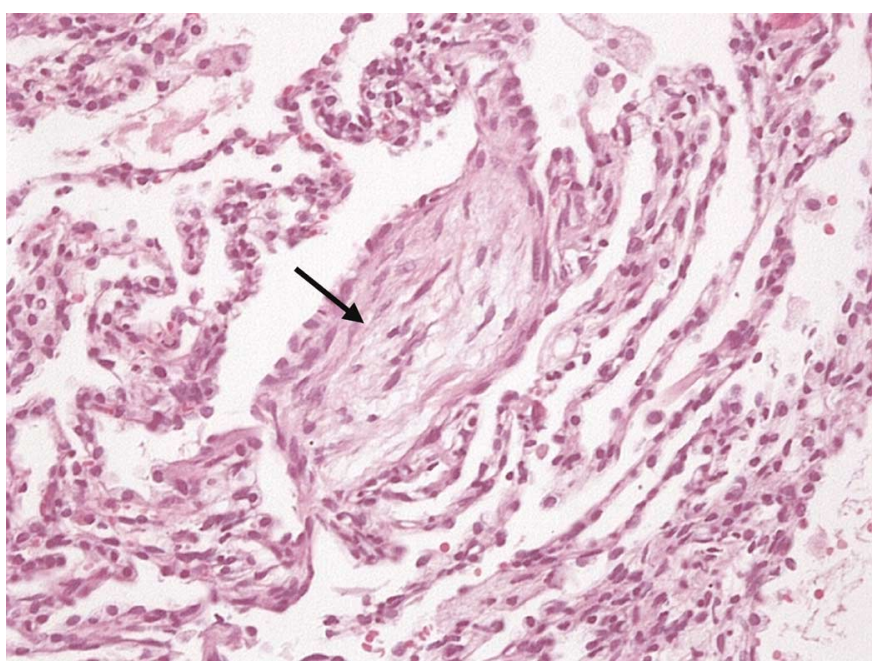

Abb 4 Alveole mit zellreichem Bindegewebspfropf, HE-Färbung $100 \mathrm{fach}$.

Monate. Dabei kam es meist bereits nach wenigen Tagen zu einer deutlichen klinischen Besserung. Rezidive wurden in der bisherigen Beobachtungszeit von 24 Monaten nicht beobachtet.

\section{Literatur}

${ }^{1}$ Prakash UBS. Radiation-induced injury in the nonirradiated lung. Eur Respir J 1999; 13: 715-717

${ }^{2}$ Cherniack RM, Abrams J, Kalica AR. Pulmonary disease associated with breast cancer therapy. Am J Respir Crit Care Med 1994; 150: $1169-1173$

${ }^{3}$ Davis SD, Yankelevitz DF, Henschke CL. Radiation effects on the lung: clinical features, pathology, and imaging findings. Am J Roentgenol 1992; 159: 1157 - 1164

${ }^{4}$ Ball D, Sephton R, Irving L et al. Radiation pneumonitis: generalised lung chages detected by radionuclide imaging following focal lung irradiation. Australes Radiol 1992; 36: $142-143$

${ }^{5}$ Roberts CM, Foulcher E, Zaunders JJ et al. Radiation pneumonitis: a possible lymphocytic-mediated hypersensitivity reaction. Ann Intern Med 1993; 118: 696 - 700

${ }^{6}$ Martin C, Romero S, Sanchez-Paya J et al. Bilateral lymphocytic alveolitis: a common reaction after unilateral thoracic radiation. Eur Respir J 1999; 13: 727-732

${ }^{7}$ Arbetter KR, Prakash UBS, Tazelaar HD et al. Radiation-induced pneumonitis in the "non-irradiated" lung. Mayo Clin Proc 1999; 74: 27 - 36

${ }^{8}$ Hallahan DE, Spriggs DR, Beckett MA et al. Increased tumor necrosis factor mRNA after cellular exposure to ionizing irradiation. Proc Natl Acad Sci USA 1989; 86: 10104-10107

${ }^{9}$ Takigawa N, Segawa Y, Saeki T et al. Bronchiolitis obliterans organizing pneumonia syndrom in breast-conserving therapy for early breast cancer: radiation-induced lung toxicity. Int J Radiat Oncol Biol Phys (United States) Okt 1 2000; 48 (3): $751-755$

${ }^{10}$ Crestani B, Valeyre D, Roderi I. Bronchiolitis obliterans organizing pneumonia syndrome primed by radiation therapy to the breast. Am J Respir Crit Care Med 1998; 158: 1929-1935 